\title{
COLLECTIVE EVIDENCE FOR INVERSE COMPTON EMISSION FROM EXTERNAL PHOTONS IN HIGH-POWER BLAZARS
}

\author{
Eileen T. Meyer ${ }^{1}$, Giovanni Fossati ${ }^{1}$, Markos Georganopoulos ${ }^{2,3}$, and Matthew L. Lister ${ }^{4}$ \\ ${ }^{1}$ Department of Physics and Astronomy, Rice University, Houston, TX 77005, USA \\ ${ }^{2}$ Department of Physics, Joint Center for Astrophysics, University of Maryland Baltimore County, 1000 Hilltop Circle, Baltimore, MD 21250, USA \\ ${ }^{3}$ NASA Goddard Space Flight Center, Code 660, Greenbelt, MD 20771, USA \\ ${ }^{4}$ Department of Physics, Purdue University, 525 Northwestern Avenue, West Lafayette, IN 47907, USA \\ Received 2012 March 22; accepted 2012 May 3; published 2012 May 18
}

\begin{abstract}
We present the first collective evidence that Fermi-detected jets of high kinetic power $\left(L_{\text {kin }}\right)$ are dominated by inverse Compton emission from upscattered external photons. Using a sample with a broad range in orientation angle, including radio galaxies and blazars, we find that very high power sources $\left(L_{\mathrm{kin}}>10^{45.5} \mathrm{erg} \mathrm{s}^{-1}\right)$ show a significant increase in the ratio of inverse Compton to synchrotron power (Compton dominance) with decreasing orientation angle, as measured by the radio core dominance and confirmed by the distribution of superluminal speeds. This increase is consistent with beaming expectations for external Compton (EC) emission, but not for synchrotron self-Compton (SSC) emission. For the lowest power jets $\left(L_{\mathrm{kin}}<10^{43.5} \mathrm{erg} \mathrm{s}^{-1}\right)$, no trend between Compton and radio core dominance is found, consistent with SSC. Importantly, the EC trend is not seen for moderately high power flat spectrum radio quasars with strong external photon fields. Coupled with the evidence that jet power is linked to the jet speed, this finding suggests that external photon fields become the dominant source of seed photons in the jet comoving frame only for the faster and therefore more powerful jets.
\end{abstract}

Key words: galaxies: active - quasars: general - radiation mechanisms: non-thermal

Online-only material: color figures

\section{INTRODUCTION}

The relativistic jets of radio-loud active galactic nuclei (AGNs) are copious gamma-ray emitters, as was first discovered by EGRET (Hartman et al. 1992), and confirmed by the Fermi Large Area Telescope (LAT), which has associated over 800 sources with radio-loud AGNs in the second catalog (2LAC; Ackermann et al. 2011). Most of these are blazars, seen with the jet axis along the line of sight, though Fermi has also detected the jets of several radio galaxies (RGs; Abdo et al. 2010; Kataoka et al. 2011), which are misaligned blazars under the standard unification scheme (Urry \& Padovani 1995).

The lower-energy peak in the jet spectrum is well understood as synchrotron emission from relativistic electrons in the jet. The high-energy component, peaking from $\mathrm{X}$-ray to $\mathrm{TeV}$ energies, is attributed to photons upscattered by the same relativistic electrons to higher energies via the inverse Compton (IC) process (see Böttcher 2007 for a review). These photons could arise from the jet synchrotron emission (synchrotron selfCompton, or SSC, emission; Maraschi et al. 1992; Marscher \& Travis 1996) or from external sources such as the accretion disk (Dermer et al. 1992), broad-line region (BLR; Sikora et al. 1994), or molecular torus (MT; Błażejowski et al. 2000; Sikora et al. 2009), i.e., external Compton (EC) emission. Identifying the IC emission mechanism is a diagnostic for the location of the gamma-ray-emitting region, currently an open issue (e.g., Agudo et al. 2011). However, the spectral energy distributions (SEDs) of individual sources are rarely sufficiently constraining of the IC mechanism due to the number of free parameters (Sikora et al. 1997).

The beaming pattern (how apparent luminosity changes with orientation) is different for EC and SSC emission. Thus, a collective study using a sample of sources at different orientations can be used to identify the gamma-ray emission mechanism. In this Letter, we discuss the effect of jet power and orientation on the observed IC power in view of the recent suggestion of a dichotomy in radio-loud AGNs (Section 2). We then show that the collective beaming pattern for a subset of high-power sources appears to support EC models rather than SSC for this population (Section 3), and discuss the implications for the gamma-ray emission region.

\section{A DICHOTOMY IN RADIO-LOUD AGNs}

\subsection{The Synchrotron Plane}

Using a large sample of jets, we recently found evidence that radio-loud AGNs form two populations in the plane of synchrotron peak luminosity $\left(L_{\mathrm{p}, \text { syn }}\right)$ versus peak frequency $\left(v_{\mathrm{p}, \mathrm{syn}}\right.$; Meyer et al. 2011, hereafter M11). A population of "weak" jets consists of sources with low $L_{\mathrm{p} \text {,syn }}$ which appear to be most aligned at high $v_{\mathrm{p} \text {,syn }}\left(\sim 10^{17} \mathrm{~Hz}\right)$ and trace out a shallow track on the plane as they become less aligned (dropping more in $v_{\mathrm{p} \text {,syn }}$ than $L_{\mathrm{p} \text {,syn }}$ ). A separate population of "strong" jets with higher $L_{\mathrm{p}, \mathrm{syn}}$ and $v_{\mathrm{p}, \mathrm{syn}} \lesssim 10^{15} \mathrm{~Hz}$ appear to drop rapidly in luminosity with misalignment (as measured by radio core dominance). Importantly, the weak jets exhibit jet kinetic powers below $10^{44.5} \mathrm{erg} \mathrm{s}^{-1}$ while all sources with $L_{\mathrm{kin}}>10^{44.5} \mathrm{erg} \mathrm{s}^{-1}$ are on the strong-jet branch. The weak/strong divide in the synchrotron plane, which we associate with the morphological dichotomy in Fanaroff-Riley (FR) type 1 and 2 RGs, may also be mapped to a critical transition in accretion efficiency (Ghisellini \& Celotti 2001; Ghisellini et al. 2009a; Georganopoulos et al. 2011).

\subsection{The Blazar Envelope at High Energies}

In light of the dichotomy discussed above, we present in Figure 1 the total LAT-band luminosity ${ }^{5}$ (a proxy for the IC

\footnotetext{
$5 \quad L_{100 \mathrm{MeV}-100 \mathrm{GeV}}=4 \pi d_{L}^{2} S_{\gamma} /(1+z)^{1-\alpha}$ for power-law sources, where $S_{\gamma}$ is the catalog-integrated energy flux and $\alpha=\Gamma-1$ using the published photon index $\Gamma$. For sources fit with $\log$-parabolic spectra, the integrated luminosity is calculated from the $2 \mathrm{LAC}$ fitted values with a $k$-correction.
} 


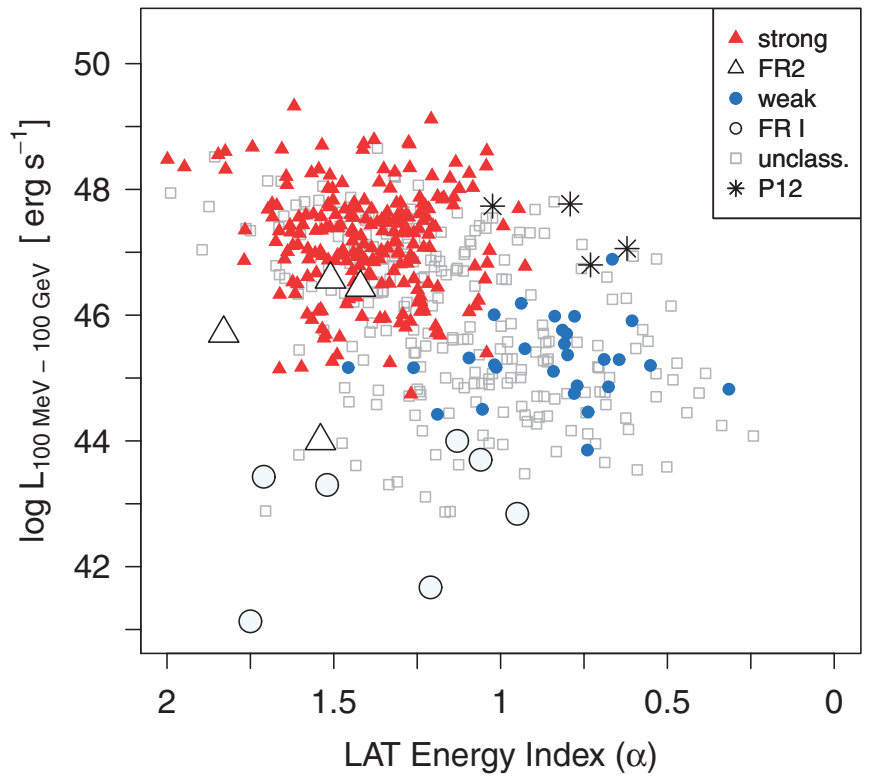

Figure 1. This plot of total LAT-band luminosity vs. gamma-ray energy index $\alpha$ (approximating $L_{\mathrm{p}, \text { IC }}$ vs. $v_{\mathrm{p}, \text { IC }}$ ) is the high-energy analog of the synchrotron plane discussed in M11. Blazars have been divided based on the luminosity and location of their synchrotron peaks into strong-jet and weak-jet blazars as described in the text. Those without $v_{\mathrm{p} \text {,syn }}$ and $L_{\mathrm{p} \text {,syn }}$ are "unclassified." FR2 RGs appear to have similar $v_{\mathrm{p} \text {, syn }}(\operatorname{similar} \alpha)$ to the strong-jet blazars while FR1 RGs have much lower values of $v_{\mathrm{p} \text {,syn }}$ (higher $\alpha$ ) relative to the weak-jet blazars with which they are associated. The FR1 would be detectable by Fermi even at much harder $\alpha$ (see Ackermann et al. 2011, Figure 15). The region at upper right remains empty despite recent claims of high-power, high-peak sources (shown as black stars; Padovani et al. 2012), matching what has been found in the synchrotron plane.

(A color version of this figure is available in the online journal.)

peak luminosity, $L_{\mathrm{p}, \mathrm{IC}}$ ), versus the LAT-band energy index $\alpha$ (a proxy for peak frequency, $v_{\mathrm{p}, \mathrm{IC}}$ ) for the entire Fermi-detected sample of radio-loud AGNs of known redshift (data taken from the 2LAC). Sources with higher $v_{\mathrm{p}, \mathrm{IC}}$ will have harder (smaller) values of $\alpha$, thus in the figure $v_{\mathrm{p}, \mathrm{IC}}$ increases to the right.

An empty region at upper right appears analogous to that seen in the synchrotron plane. Giommi et al. (2012) have suggested that this is a selection effect due to a lack of redshifts for highfrequency-peaked, high-luminosity sources, and Padovani et al. (2012) discuss four such candidates. However, these candidates do not cross into the upper-right region in Figure 1 (black stars). These interesting sources appear to exhibit $v_{\mathrm{p} \text {,syn }}$ at or above $\sim 10^{15} \mathrm{~Hz}$; however, the SED sampling is sparse, and it is difficult to rule out an alternative explanation for the soft X-ray spectra such as an extra emission component (see, e.g., the case of BL Lacertae; Raiteri et al. 2010). Importantly, for the several hundred Fermi sources lacking identifications or redshifts, nearly all ( $>99 \%)$ have soft spectra $(\alpha>1)$ or would require redshifts $>2$ or higher to cross into this region, making it unlikely to be empty due to selection effects.

The blazars in Figure 1 have been divided in an approximate way based on their synchrotron spectra into strong and weak jets. We take the former to be those defined by $L_{\mathrm{p}, \mathrm{syn}}>10^{45} \mathrm{erg} \mathrm{s}^{-1}$, $v_{\mathrm{p} \text {,syn }}<10^{15} \mathrm{~Hz}$, as well as flat spectrum radio quasar (FSRQ) type sources outside this area. For the weak jets, we take all sources outside this area, minus any FSRQ. While very rare cases of FR1-like (e.g., weak) FSRQs exist, their occurrence is negligible in the bright Fermi-detected population considered here (see, e.g., Kharb et al. 2010 and references therein). The 11 Fermi-detected RGs are also shown (data from Abdo et al. 2010).

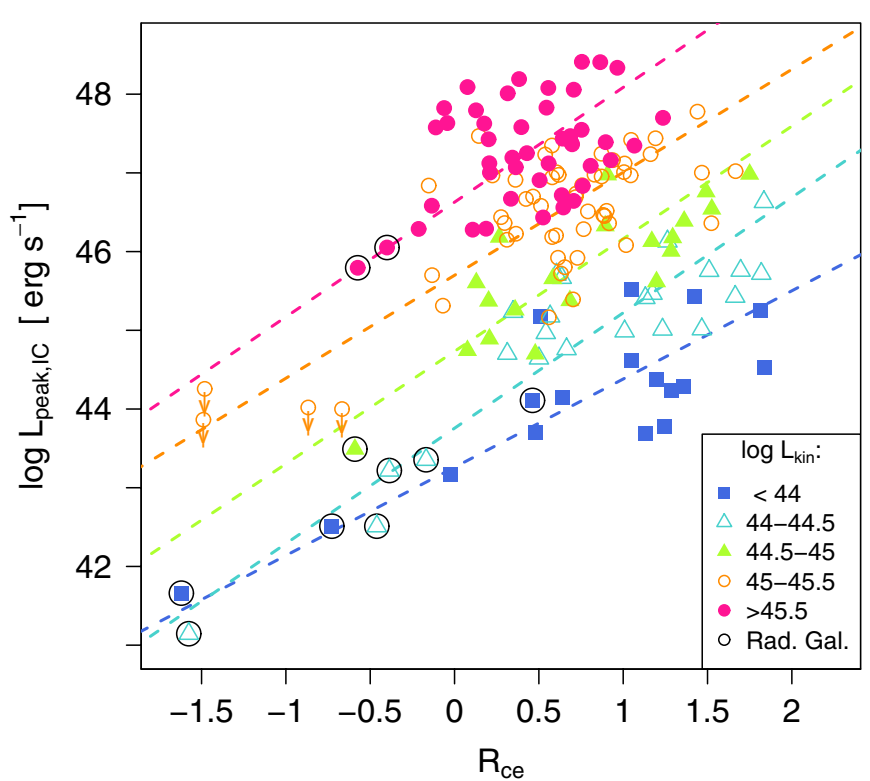

Figure 2. Estimated IC peak luminosity $\left(L_{\mathrm{p}, \text { IC }}\right)$ vs. radio core dominance $\left(R_{\mathrm{ce}}\right)$ for 145 blazars and 10 RGs (circled) detected by Fermi. The correlation between $L_{\mathrm{p}, \text { IC }}$ and $R_{\mathrm{ce}}$ becomes clear when binning by $L_{\mathrm{kin}}$. The OLS bisector fits to sources grouped by $L_{\text {kin }}$ have slopes $1.1,1.5,1.4,1.3,1.5( \pm 0.1$; Pearson's $R$ values $0.81,0.89,0.82,0.37,0.44$ ) beginning with the lowest bin. For an EC emission model, these slopes are predicted to be higher $(>1.7)$; however, for the high-power sources, the lack of Fermi detections for misaligned (i.e., low $R_{\text {ce }}$ ) sources will affect the apparent slope.

(A color version of this figure is available in the online journal.)

The FR1 in Figure 1 have much lower $v_{\mathrm{p}, \mathrm{IC}}$ (larger $\alpha$ ) compared with the weak-jet sources, while there is little difference between $\alpha$ for FR2 and strong jets, tentatively matching the different misalignment paths for these populations that were found in the synchrotron plane.

\subsection{The Importance of Jet Kinetic Power}

In M11, we showed that $L_{\mathrm{kin}}$ is an important parameter in classifying radio-loud AGNs. We have selected from the $2 \mathrm{LAC}$ a subset of 152 blazars (as well as the detected RGs) with estimates of the $300 \mathrm{MHz}$ isotropic lobe emission, which is scaled to estimate $L_{\text {kin }}$ as in M11. For 46 sources with adequate coverage of the high-energy SED, we estimated $L_{\mathrm{p}, \mathrm{IC}}$ from a two-sided parabolic fit to the 2LAC data in combination with X-ray data taken from $\mathrm{NED}^{6}$ and/or Swift/BAT (Cusumano et al. 2010). For an additional 106 sources, $L_{\mathrm{p}, \mathrm{IC}}$ is scaled from the rest-frame luminosity at $1 \mathrm{GeV}$ estimated from the $2 \mathrm{LAC}$ fitted spectrum: $\log L_{\mathrm{p}, \mathrm{IC}}=\log L_{1 \mathrm{GeV}}+\eta(\alpha-1)^{2}$, where $\eta=$ 1.5 if $\alpha>1, \eta=5$ otherwise. The difference between the two estimates is typically less than 0.2 decades in $\log L$.

We use the radio core dominance $R_{\mathrm{ce}} \equiv \log \left(L_{\text {core }} / L_{\text {ext }}\right)$ as a tracer of the orientation angle, where the core luminosity is measured at $1.4 \mathrm{GHz}$ and the extended at $300 \mathrm{MHz}$ (see M11). As discussed in M11, the absolute normalization between orientation angle and $R_{\text {ce }}$ depends on $L_{\text {kin }}$, with $R_{\text {ce }}$ decreasing with increasing orientation angle for a given $L_{\text {kin }}$.

In Figure 2 we show the 145 sources which have a known redshift. $L_{\mathrm{p}, \mathrm{IC}}$ is positively correlated with both $R_{\mathrm{ce}}$ and $L_{\mathrm{kin}}$. The OLS bisector fits to the combined blazar-RG sample shown have slopes of $1.1,1.5,1.4,1.3,1.5( \pm 0.1)$ from the lowest to highest bin in $L_{\text {kin }}$. The correlation between $L_{\mathrm{p}, \mathrm{IC}}$ and $R_{\mathrm{ce}}$ in

\footnotetext{
6 NASA Extragalactic Database: http://ned.ipac.caltech.edu/
} 
Table 1

Predicted Correlation Slope Values

\begin{tabular}{|c|c|c|c|c|c|c|}
\hline \multicolumn{3}{|c|}{ Index Values } & \multicolumn{2}{|c|}{$\mathrm{SSC}$} & \multicolumn{2}{|c|}{$\mathrm{EC}$} \\
\hline$p_{r}$ & $p_{\text {syn }}$ & $p_{\gamma}$ & $L_{\gamma}-R_{\mathrm{ce}}$ & $R_{\mathrm{p}}-R_{\mathrm{ce}}$ & $L_{\gamma}-R_{\mathrm{ce}}$ & $R_{\mathrm{p}}-R_{\mathrm{ce}}$ \\
\hline 3 & 3 & 3 & $1.1-1.3$ & 0 & $1.7-2$ & $0.6-0.7$ \\
\hline 2 & 3 & 3 & $1.6-2$ & 0 & $2.4-3$ & $0.8-1$ \\
\hline 2 & 2 & 3 & $1.6-2$ & 0 & $2.4-3$ & $1.2-1.5$ \\
\hline 2 & 2 & 2 & $1.2-1.5$ & 0 & $1.7-2.5$ & $0.8-1$ \\
\hline
\end{tabular}

each group is significant and positive ( $R$ values from 0.37 to $0.89)$; the apparent gamma-ray output of a blazar is therefore a strong function of both $L_{\text {kin }}$ and the orientation angle.

\subsection{Emission Mechanisms and Their Beaming Patterns}

For both synchrotron and SSC, the beaming pattern is $L=$ $L^{\prime} \delta^{p+\alpha}$ (Dermer 1995), where $L$ assumes isotropic emission in the galaxy frame, $L^{\prime}$ is the solid-angle integrated luminosity in the comoving jet frame, $\delta$ is the Doppler factor, and $\alpha$ is the spectral index. For the exponent $p+\alpha, p=3$ for a "moving blob," or $p=2$ for a stationary feature in a continuous jet (Lind \& Blandford 1985), with the possibility of different values for different emitting regions. For the EC case, the pattern is $L_{\mathrm{EC}}=L_{\mathrm{EC}}^{\prime} \delta^{p+1+2 \alpha}$ (Dermer 1995; Georganopoulos et al. 2001).

The slope of $L_{\mathrm{p}, \text { IC }}$ versus $R_{\mathrm{ce}}$ depends on the gamma-ray emission process. From the synchrotron beaming,

$$
R_{\text {ce }} \equiv \log \left(L_{\text {core }} / L_{\text {ext }}\right)=\left(p_{r}+\alpha_{r}\right) \log \delta+c_{1},
$$

where the factors $c_{n}$ depend on the unbeamed luminosity and jet power, but do not affect the slope. We take $\alpha=1$ for the peaks, and eliminating $\delta$ we obtain:

$$
\log L_{\text {peak }}=\left(\frac{b}{p_{r}+\alpha_{r}}\right) R_{\mathrm{ce}}+c_{2},
$$

where $b=1+p_{\gamma}(\mathrm{SSC})$ or $b=3+p_{\gamma}(\mathrm{EC})$. For reference we list in Table 1 the expected slopes for various cases of $p$ values assuming typical values of $\alpha_{r}(0-0.5)$. The slopes in Figure 2 appear to be more consistent with SSC under a $p_{\gamma}, p_{r}=3$ or $p_{\gamma}, p_{r}=2$ scenario. However, the lack of Fermi-detected misaligned FR2 sources (particularly with $R_{\text {ce }}<-0.5$ ) is likely to affect the slope for the two highest bins in $L_{\text {kin }}$ in Figure 2; we show for reference the upper limits to $L_{\mathrm{p}, \mathrm{IC}}{ }^{7}$ for four RGs in the M11 sample. We take the rightmost of these points as the most constraining: including it in the fit for the $\log L_{\text {kin }}=45-45.5$ bin increases the slope to $1.5 \pm 0.2$, and for the highest bin to $1.7 \pm 0.3$. These slopes, and our assumption that the Lorentz factor $(\Gamma)$ of the gamma-ray-emitting plasma is similar to that in radio, are discussed below.

\section{THE COMPTON DOMINANCE IN FERMI BLAZARS}

\subsection{EC in Powerful FSRQ}

For sources with a high-energy component dominated by $\mathrm{SSC}$, the Compton dominance $\left(R_{\mathrm{p}}\right)$ should remain constant over all orientations (no correlation between $R_{\mathrm{p}}$ and $R_{\text {ce }}$ expected). For the EC case,

$$
R_{p} \equiv \log \left(L_{\mathrm{EC}} / L_{S}\right)=\left(p_{\gamma}-p_{\mathrm{syn}}+2\right) \log \delta+c_{3}
$$

\footnotetext{
7 From the flux limit $(>100 \mathrm{MeV})$ : $\log f_{100}=-8.3+(5 / 6)(\alpha-1.2)$, with average $\alpha=1.3$ for our sample and $L_{\mathrm{p}, \mathrm{IC}}$ scaled from $L_{1 \mathrm{GeV}}$ (Section 2.3).
}

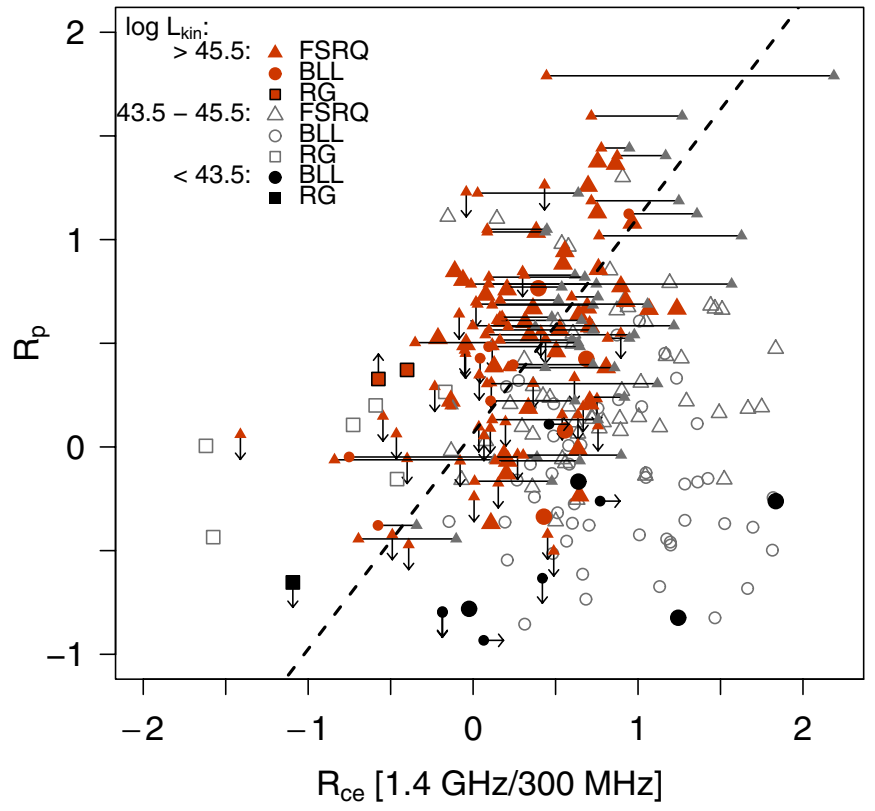

Figure 3. Compton dominance $\left(R_{\mathrm{p}}\right)$ vs. radio core dominance $\left(R_{\mathrm{ce}}\right)$. A positive correlation is seen for the most powerful sources (shaded), as predicted for EC emission. The slope of the OLS bisector fit to the shaded points is $1.1 \pm 0.1$, though the presence of the upper limits on $R_{\mathrm{p}}$ and lower limits on $R_{\mathrm{ce}}$ (range shown by black lines) makes this a lower limit on the real slope. Weak jets (black points) as well as moderately powerful FSRQ (open triangles) show no trend, as expected for simple SSC.

(A color version of this figure is available in the online journal.)

Using Equation (1) we have the relation

$$
R_{p}=\left(\frac{p_{\gamma}-p_{\mathrm{syn}}+2}{p_{r}+\alpha_{r}}\right) R_{c e}+c_{4} .
$$

As shown in Table 1, we expect a correlation with a slope from 0.6 to 1.5 under the simplest assumptions for sources emitting gamma rays by EC.

Figure 3 shows $R_{\mathrm{p}}$ versus $R_{\text {ce }}$ for the sample discussed in Section 2.3. Sources are divided into broad bins of $L_{\text {kin }}$. These bins include a mix of sources in terms of optical type (FSRQ or BL Lac); however all the very high power (VHP, $L_{\text {kin }}>10^{45.5}$ ) are "strong-jet" types in our classification due to their $L_{\text {kin }}$ and position in the synchrotron plane, including many apparent $\mathrm{BL}$ Lac objects, which likely suffer dilution of their broad lines by the jet emission (Georganopoulos \& Marscher 1998; Ghisellini et al. 2011). For the moderate-power sources, the division into strong/weak roughly follows the FSRQ/BL Lac divide.

No trend between $R_{\mathrm{p}}$ and $R_{\mathrm{ce}}$ is evident for blazars overall. However, when VHP sources are selected, a positive correlation emerges. We also show the upper limits on $R_{\mathrm{p}}$ for VHP sources from the sample of M11 not detected by Fermi ${ }^{8}$ VHP sources from $2 \mathrm{LAC}$ and M11 with upper limits on $L_{\mathrm{ext}}{ }^{9}$ are also shown; because of the binning on $L_{\text {kin }}$ which is scaled from $L_{\text {ext }}$, we also show as connected gray points the maximum $R_{\mathrm{ce}}$ such that $L_{\text {kin }}>10^{45.5} \mathrm{erg} \mathrm{s}^{-1}$. The OLS bisector fit through all VHP points (including upper/lower limits) gives a slope of $1.1 \pm 0.1$; this is a lower limit since most of the $R_{\mathrm{p}}$ upper limits are on the lower half of the correlation. A sub-sample of sources with

\footnotetext{
8 See previous footnote, with index $\alpha$ estimated from the relation for detected sources: $\alpha=-0.2 \times v_{\mathrm{p}, \text { syn }}+3.9$.

9 Estimated at $300 \mathrm{MHz}$ from the lowest-frequency SED point with spectral index $\alpha=1.2$.
} 

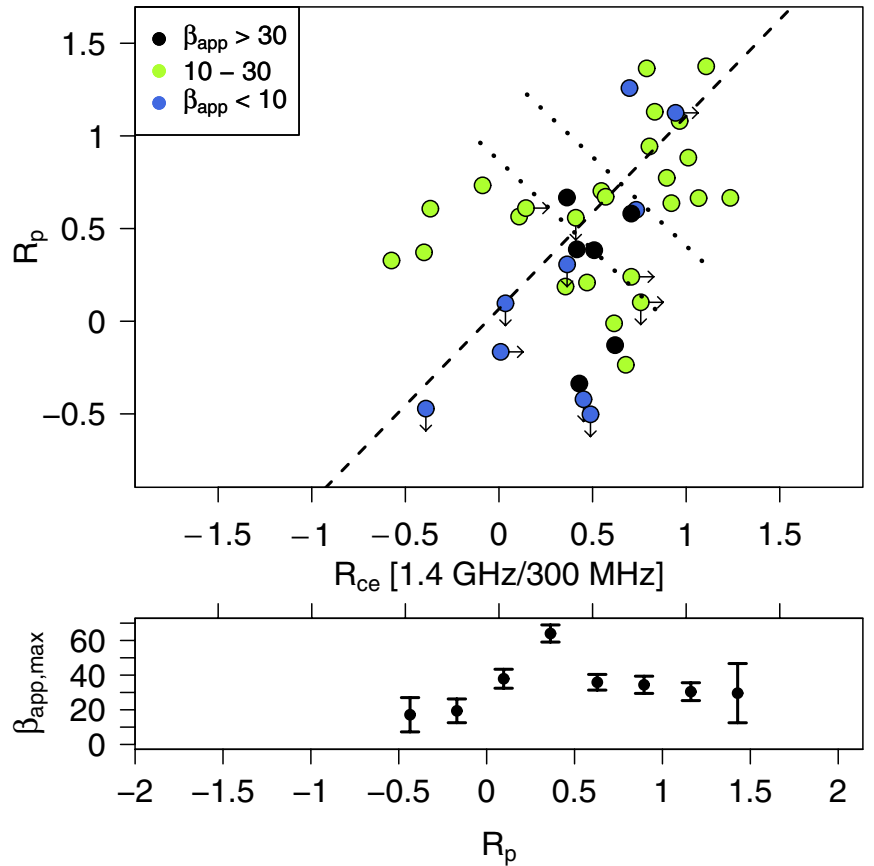

Figure 4. Upper: the same plot as Figure 3, for high-power sources $\left(L_{\mathrm{kin}}>\right.$ $10^{45.5} \mathrm{erg} \mathrm{s}^{-1}$ ) with measurements of maximum apparent jet speed $\left(\beta_{\mathrm{app}}\right)$. Values are taken from Jorstad et al. (2001, 2005), Kellermann et al. (2004), Lister et al. (2009), and Kharb et al. (2010). The appearance of low $\beta_{\text {app }}$ sources with high core and Compton dominance supports the interpretation that these sources are aligned very close to the line of sight. Lower: sources are binned along the shown correlation (running perpendicular to the dashed line in steps of 0.25 of $R_{\text {ce }}$ as illustrated by the two dotted lines), and the maximum $\beta_{\text {app }}$ is estimated. The increase in $\beta_{\mathrm{app}, \text { max }}$ from lower $R_{\mathrm{ce}} / R_{\mathrm{p}}$ toward a maximum, followed by a decrease toward the highest values is qualitatively as expected if $R_{\text {ce }}$ and $R_{\mathrm{p}}$ increase with alignment.

(A color version of this figure is available in the online journal.)

$R_{\text {ce }}$ values contemporaneous to Fermi, calculated from the average $15 \mathrm{GHz}^{10}$ flux over the time frame of the $2 \mathrm{LAC}$ as measured by the Owens Valley Radio Observatory (OVRO; Richards et al. 2011), gives an identical slope.

The non-zero slope suggests EC emission in VHP sources; however, when taken together the slopes in Figures 2 and 3 are inconsistent with a simple EC scenario, as seen from Table 1. We have assumed that $\delta$ is the same for all energies; if the radioemitting plasma is slower, then the expected slopes above will increase from the quoted ranges for both figures. Assuming truly higher slopes for the VHP sources in Figure 2, concordance can be achieved with a general $p=3$ model with a slower $\Gamma$ in the radio.

\subsection{The Test of Superluminal Motions}

As shown by Lister \& Marscher (1997), a substantial number of sources are expected to be seen at orientation angles smaller than that of $\theta=1 / \Gamma$ which maximizes the superluminal speed $\beta_{\text {app }}$. We therefore expect the highest $\beta_{\text {app }}$ values at $R_{\mathrm{p}}\left(R_{\mathrm{ce}}\right)$ values less than the maximum. Indeed if the highest $R_{\mathrm{p}}\left(R_{\mathrm{ce}}\right)$ sources are most aligned (within $1 / \Gamma$ ), their $\beta_{\text {app }}$ values should be relatively small (noting that these may be underrepresented as their $\beta_{\text {app }}$ are difficult to measure). In Figure 4 (upper panel) the VHP sources are plotted as in Figure 3, colored according to $\beta_{\text {app }}$. The highest $\beta_{\text {app }}$ are observed in the middle of the correlation, as expected. This is also seen in the lower panel,

\footnotetext{
10 Scaled to $1.4 \mathrm{GHz}$ by -1.2 in $\log L$.
}

where we have estimated the maximum $\beta_{\text {app }}$ in bins of width 0.25 in $R_{\text {ce }}$, running parallel to the correlation line. ${ }^{11}$

\subsection{Why is EC Only Apparent in Powerful Jets?}

What is apparently depicted in Figure 3 is that strong jets at high $L_{\text {kin }}$ are EC dominated, while lower $L_{\text {kin }}$ strong jets are not. However, the typical error in $\log L_{\text {kin }}$ of 0.7 is a significant factor, as it may be the case that the restriction $L_{\text {kin }}>10^{45.5} \mathrm{erg} \mathrm{s}^{-1}$ is simply that which is high enough to avoid any contamination with SSC sources. Thus the true boundary $L_{\text {kin }}$ for the SSC to EC transition is probably lower than $10^{45.5}$ (indeed, a few FSRQ of moderate $L_{\text {kin }}$ are cospatial in Figure 3 with the VHP sources). However, our findings imply that (1) VHP sources are dominated by EC and (2) many lower $L_{\text {kin }}$ strong jets appear at a similar (low) $R_{\mathrm{p}}$ regardless of $R_{\mathrm{ce}}$, suggesting that EC is not important for some part of the population.

Which emission mechanism dominates is related to how fast the flow is: assuming the gamma-ray emission of strong jets is inside the BLR or MT, the comoving energy density of the external photon field is $\Gamma^{2} U_{\text {ext }}$. The synchrotron energy density is $U_{\mathrm{s}}=L_{\mathrm{s}} /\left(4 \pi c^{3} t_{\mathrm{var}}^{2} \Gamma^{6}\right)$, where $t_{\mathrm{var}}$ is the observed variability timescale in hours, $L_{\mathrm{s}}$ is the synchrotron luminosity, and we have assumed $\delta=\Gamma$. EC will dominate over SSC provided $\Gamma^{2} U_{\text {ext }}>U_{\mathrm{s}}$, or for $\Gamma$ greater than a transition value

$$
\Gamma_{\mathrm{tr}}=16.2\left(\frac{L_{\mathrm{s}} / 10^{47}}{\left(t_{\mathrm{var}} / 6\right)^{2}\left(U_{\mathrm{ext}} / 10^{-4}\right)}\right)^{\frac{1}{8}} .
$$

Typical estimates of $U_{\text {ext }}$ in the BLR and MT differ by $\sim 100$

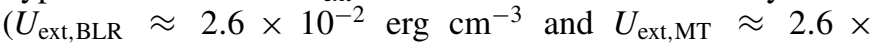
$10^{-4}$ erg $\mathrm{cm}^{-3}$; Ghisellini et al. 2009b). In the MT we have $\Gamma_{\text {tr,MT }} \sim 14.4$, higher by a factor of $100^{1 / 8}=1.8$ than in the BLR, where $\Gamma_{\text {tr,BLR }} \sim 8.1$.

However, very long baseline interferometry studies indicate that $\Gamma \gtrsim 10$ for most FSRQ (e.g., Jorstad et al. 2005); if the $\mathrm{GeV}$ emission site is in the BLR, it is difficult to explain the lack of EC signature for some strong jets. It has also been found that sources with higher $L_{\text {kin }}$ produce on the average faster superluminal motions (Kharb et al. 2010). The connection between $L_{\text {kin }}$ and $\Gamma$, and an emitting region in the $M T$, can then explain a transition to EC at high $L_{\mathrm{kin}}$ : strong jets at lower $L_{\mathrm{kin}}$ are also slower and as long as $\Gamma<\Gamma_{\text {tr }}$ they are SSC emitters, exhibiting a Compton dominance independent of radio core dominance. As $L_{\text {kin }}$ increases, $\Gamma$ also increases and once $\Gamma>\Gamma_{\text {tr }}$, the photon field of the MT begins to dominate, producing a Compton dominance that increases with increasing radio core dominance, as seen in Figure 3 . If we adopt longer $t_{\mathrm{var}}$, both $\Gamma_{\text {tr,BLR }}$ and $\Gamma_{\text {tr,MT }}$ decrease, requiring that essentially all strong jets are EC emitters, which our data do not support. On the other hand, with a shorter $t_{\mathrm{var}} \sim 1 \mathrm{hr}$ (see, e.g., Foschini et al. 2011) we obtain $\Gamma_{\mathrm{tr}}=12.7$ for the BLR and $\Gamma_{\mathrm{tr}}=22.5$ for the MT, making either location plausible.

\section{CONCLUSIONS}

The gamma-ray luminosities of Fermi-detected radio-loud AGNs have been shown to depend strongly on both $L_{\text {kin }}$ and the orientation angle. We find the first collective evidence for

\footnotetext{
11 The estimator for $\beta_{\text {app, max }}$ is derived from the order statistic $Y_{n}=\max \left(\beta_{\text {app }}\right)$ for a bin of size $n$ using the estimator for the unknown upper bound of a uniform distribution $\beta_{\mathrm{app}, \max }=Y_{n}(n+1) / n$ with variance $\beta_{\mathrm{app}, \max }^{2} /\left(n^{2}+2 n\right)$ (e.g., Casella \& Berger 2001).
} 
EC emission in high-power jets $\left(L_{\text {kin }}>10^{45.5} \mathrm{erg} \mathrm{s}^{-1}\right)$ : as can be seen in Figure 3, the Compton dominance of these sources increases with radio core dominance, a measure of orientation. This requires that the beaming pattern of the gamma-ray emission is more focused than that of synchrotron, as is the case for EC scattering. A confirmation that $R_{\mathrm{p}}$ increases with decreasing orientation angle comes from the fact that the apparent superluminal speeds are observed to increase along the correlation to a maximum at moderate values of $R_{\mathrm{p}}$, before decreasing toward the highest values (Figure 4), as expected since we anticipate a significant number of sources at angles smaller than the maximum superluminal speed angle $1 / \Gamma$. The fact that strong jets of lower $L_{\text {kin }}$ do not show an increase of Compton dominance with alignment suggests that their gamma-ray emission is due to SSC. Because more powerful jets appear to be faster (Kharb et al. 2010), a transition to EC at high $L_{\text {kin }}$ can be explained by a transition Lorentz factor above which the external photons dominate.

E.M. and G.F. acknowledge support from NASA Fermi grants NNX11AO15G and NNX10AO42G, Swift grant NNX09AR04G, and XMM grant NNX06AE92G. M.G. acknowledges support from NASA ATFP grant NNX08AG77G and Fermi grant NNX12AF01G. M.L. and the MOJAVE project is supported under National Science Foundation grant 0807860-AST.

\section{REFERENCES}

Abdo, A. A., Ackermann, M., Ajello, M., et al. 2010, ApJ, 720, 912

Ackermann, M., Ajello, M., Allafort, A., et al. 2011, ApJ, 743, 171

Agudo, I., Marscher, A. P., Jorstad, S. G., et al. 2011, ApJ, 735, L10

Błażejowski, M., Sikora, M., Moderski, R., \& Madejski, G. M. 2000, ApJ, 545, 107
Böttcher, M. 2007, Ap\&SS, 309, 95

Casella, G., \& Berger, R. 2001, Statistical Inference (Pacific Grove, CA: Duxbury Press)

Cusumano, G., La Parola, V., Segreto, A., et al. 2010, A\&A, 510, A48

Dermer, C. D. 1995, ApJ, 446, L63

Dermer, C. D., Schlickeiser, R., \& Mastichiadis, A. 1992, A\&A, 256, L27

Foschini, L., Ghisellini, G., Tavecchio, F., Bonnoli, G., \& Stamerra, A. 2011, A\&A, 530, A77

Georganopoulos, M., Kirk, J. G., \& Mastichiadis, A. 2001, ApJ, 561, 111

Georganopoulos, M., \& Marscher, A. P. 1998, ApJ, 506, 621

Georganopoulos, M., Meyer, E. T., Fossati, G., \& Lister, M. L. 2011, arXiv: 1111.4711

Ghisellini, G., \& Celotti, A. 2001, A\&A, 379, L1

Ghisellini, G., Maraschi, L., \& Tavecchio, F. 2009a, MNRAS, 396, L105

Ghisellini, G., Tavecchio, F., Foschini, L., \& Ghirlanda, G. 2011, MNRAS, 414 2674

Ghisellini, G., Tavecchio, F., \& Ghirlanda, G. 2009b, MNRAS, 399, 2041

Giommi, P., Padovani, P., Polenta, G., et al. 2012, MNRAS, 420, 2899

Hartman, R. C., Bertsch, D. L., Fichtel, C. E., et al. 1992, ApJ, 385, L1

Jorstad, S. G., Marscher, A. P., Lister, M. L., et al. 2005, AJ, 130, 1418

Jorstad, S. G., Marscher, A. P., Mattox, J. R., et al. 2001, ApJS, 134, 181

Kataoka, J., Stawarz, Ł., Takahashi, Y., et al. 2011, ApJ, 740, 29

Kellermann, K. I., Lister, M. L., Homan, D. C., et al. 2004, ApJ, 609, 539

Kharb, P., Lister, M. L., \& Cooper, N. J. 2010, ApJ, 710, 764

Lind, K. R., \& Blandford, R. D. 1985, ApJ, 295, 358

Lister, M. L., Cohen, M. H., Homan, D. C., et al. 2009, AJ, 138, 1874

Lister, M. L., \& Marscher, A. P. 1997, ApJ, 476, 572

Maraschi, L., Ghisellini, G., \& Celotti, A. 1992, ApJ, 397, L5

Marscher, A. P., \& Travis, J. P. 1996, A\&AS, 120, C537

Meyer, E. T., Fossati, G., Georganopoulos, M., \& Lister, M. L. 2011, ApJ, 740 , 98

Padovani, P., Giommi, P., \& Rau, A. 2012, MNRAS, 422, L48

Raiteri, C. M., Villata, M., Bruschini, L., et al. 2010, A\&A, 524, A43

Richards, J. L., Max-Moerbeck, W., Pavlidou, V., et al. 2011, ApJS, 194, 29

Sikora, M., Begelman, M. C., \& Rees, M. J. 1994, ApJ, 421, 153

Sikora, M., Madejski, G., Moderski, R., \& Poutanen, J. 1997, ApJ, 484, 108

Sikora, M., Stawarz, Ł., Moderski, R., Nalewajko, K., \& Madejski, G. M. 2009, ApJ, 704, 38

Urry, C. M., \& Padovani, P. 1995, PASP, 107, 803 